\title{
New WHO treatment recommendations for multidrug-resistant tuberculosis: Are we well enough prepared?
}

Katharina Kranzer ${ }^{1,2}$, Barbara Kalsdorf ${ }^{3,4,5}$, Jan Heyckendorf ${ }^{3,4,5}$, Sönke Andres ${ }^{1}$, Matthias Merker $^{4,6}$, Sabine Hofmann-Thiel ${ }^{7,8}$, Guido V. Bloemberg 9 , Harald Hoffmann ${ }^{7,8}$, Stefan Niemann ${ }^{1,4,6}$, Christoph Lange $3,4,5,10$, Florian P. Maurer $1,4, \#$

${ }^{1}$ National and WHO Supranational Reference Laboratory for Mycobacteria, Research Center Borstel, Leibniz Lung Center, Borstel, Germany

2 Department of Clinical Research, London School of Hygiene and Tropical Medicine, London, UK.

${ }^{3}$ Clinical Infectious Diseases, Research Center Borstel, Leibniz Lung Center, Borstel, Germany

${ }^{4}$ German Center for Infection Research (DZIF), Hamburg, Germany

${ }^{5}$ International Health/Infectious Diseases, University of Lübeck, Lübeck, Germany

${ }^{6}$ Experimental and Molecular Mycobacteriology, Research Center Borstel, Leibniz Lung Center, Borstel, Germany

7 SYNLAB Gauting, Gauting, Germany

${ }^{8}$ IMLred, WHO Supranational Reference Laboratory for TB, Gauting, Germany

${ }^{9}$ Institute for Food Safety and Hygiene, University of Zürich, Zürich, Switzerland

10 Department of Medicine, Karolinska Institute, Stockholm, Sweden

\# corresponding author:

PD Dr. med. Florian P. Maurer

National and WHO Supranational Reference Laboratory for Mycobacteria Research Center Borstel, Leibniz Lung Center

Parkallee 18, 23845 Borstel, Germany

fmaurer@fz-borstel.de

+49 (0) $4537-188-2110$ 


\section{To the Editor:}

The World Health Organization (WHO) recently announced extensively revised treatment recommendations for multidrug-resistant tuberculosis (MDR-TB) ${ }^{1}$. Bedaquiline (Bdq) is now categorized as a group A drug together with later generation fluoroquinolones and linezolid to be comprised in any MDR-TB regimen unless drug resistance is observed. Resistance to Bdq has been reported in up to $6 \%$ of Bdq-naïve patients. ${ }^{2}$ In addition, acquired resistance to Bdq has been described in 2015 for the first time. ${ }^{3}$

Recently, we observed two new cases of acquired resistance to Bdq in a 26-year old male from Armenia and in a 28-year old female from Russia suffering from pre-extensively and extensively drug-resistant TB, respectively. Empirical drug-regimens including Bdq were adjusted informed by second-line drug-susceptibility testing (DST) results upon referral to our specialized clinic. Increased Bdq MIC values with bacterial growth at or above the critical concentration of $1.0 \mathrm{mg} / \mathrm{L}$ were observed in follow-up isolates from both patients despite individualized therapy in accordance with current guidelines in a specialized, multidisciplinary setting.

We acknowledge that Bdq is the only drug for treatment of MDR-TB with proven efficacy demonstrated in randomized controlled trials. ${ }^{4}$ However, endorsing Bdq as core drug for the treatment of all MDR-TB patients will increase the risk for acquired resistance and transmission of Bdq-resistant strains. This risk needs to be mitigated by routine DST of Bdq and all its companion drugs. Recently, Cox and Mizrahi pointed out that $22 \%$ of the globally 6.3 million people with newly diagnosed TB in 2016 did not even have access to rifampin DST. ${ }^{5}$ Currently, Bdq DST is restricted to a small number of reference laboratories. Furthermore, there are no commercially available molecular tests for rapid genotypic DST of Bdq or the prioritized companion drugs clofazimine, linezolid, and cycloserine proposed to support Bdq and the fluoroquinolones in a standard combination regimen for MDR-TB. In the light of the new WHO recommendations, global capacities to perform DST of the prioritized drugs need to be scaledup as a matter of urgency. 


\section{References:}

1. WHO consolidated guidelines on drug-resistant tuberculosis treatment. Geneva: World Health Organization; 2019. Licence: CC BY-NC-SA 3.0 IGO

2. Villellas C, Coeck N, Meehan CJ, et al. Unexpected high prevalence of resistanceassociated Rv0678 variants in MDR-TB patients without documented prior use of clofazimine or bedaquiline. J Antimicrob Chemother [Internet] 2017;72(3):684-90. Available from: http://www.ncbi.nlm.nih.gov/pubmed/28031270

3. Bloemberg G V, Keller PM, Stucki D, et al. Acquired Resistance to Bedaquiline and Delamanid in Therapy for Tuberculosis. N Engl J Med [Internet] 2015;373(20):1986-8. Available from: http://www.ncbi.nlm.nih.gov/pubmed/26559594

4. Diacon AH, Pym A, Grobusch MP, et al. Multidrug-resistant tuberculosis and culture conversion with bedaquiline. N Engl J Med [Internet] 2014;371(8):723-32. Available from: http://www.ncbi.nlm.nih.gov/pubmed/25140958

5. Cox H, Mizrahi V. The Coming of Age of Drug-Susceptibility Testing for Tuberculosis. N Engl J Med [Internet] 2018;379(15):1474-5. Available from: http://www.ncbi.nlm.nih.gov/pubmed/30256713 